\title{
A Multifaceted Approach for Safety Design and Probabilistic Optimization
}

\author{
Po Ting Lin, ${ }^{1}$ Mark Christian E. Manuel, ${ }^{1}$ Yi-Hung Liu, ${ }^{1}$ Yu-Cheng Chou, ${ }^{2}$ Yung Ting, \\ Shian-Shing Shyu, ${ }^{3}$ Chang-Kuo Chen, ${ }^{3}$ and Chun-Lin Lee ${ }^{3}$ \\ ${ }^{1}$ Department of Mechanical Engineering, Chung Yuan Christian University, Chungli, Taoyuan County 32023, Taiwan \\ ${ }^{2}$ Institute of Undersea Technology, National Sun Yat-sen University, Kaohsiung City 80424, Taiwan \\ ${ }^{3}$ Institute of Nuclear Energy Research, Atomic Energy Council, Longtan Township, Taoyuan County 32546, Taiwan \\ Correspondence should be addressed to Po Ting Lin; potinglin@cycu.edu.tw
}

Received 13 May 2014; Accepted 2 September 2014

Academic Editor: Kang Li

Copyright (C) 2015 Po Ting Lin et al. This is an open access article distributed under the Creative Commons Attribution License, which permits unrestricted use, distribution, and reproduction in any medium, provided the original work is properly cited.

\begin{abstract}
Safety design and probabilistic optimization are fields that are widely subject to uncertainty, thus making traditional deterministic methods highly unreliable for these two fields. Popular design optimizations methods widely used for safety design and probabilistic optimization are the performance measure approach (PMA) and the performance measure approach (RIA). In a problem where the analysis is performed from an infeasible design space, a modified reliability index approach (MRIA) is employed to address some inefficiency of the traditional RIA to be able to find the optimal solutions. The PMA uses an inverse reliability analysis, which is more computationally efficient at finding the most probable design points but has been reported to have numerical instabilities on some cases. In this paper, three benchmark examples were thoroughly studied with various initial points to examine the stability and efficiency of MRIA and PMA. A hybrid reliability approach (HRA) was then presented after determining a selection factor from the optimum conditions. The proposed HRA aims to determine which of the two optimization methods would be more appropriate during the optimization processes.
\end{abstract}

\section{Introduction}

Traditional deterministic approaches for optimization of components, products, and systems have been replaced in the past decades of approaches that integrate probabilistic considerations due to the nature of the problem for safety design and probabilistic considerations. These probabilistic considerations might be allowable failure probabilities from standards [1], reported incidences of failures or satisfactions [2], production condition [3], operation condition [4], and material property deviation [5], to mention a few. Incorporating these factors in the optimization process has been the basic concept of reliability based design optimization (RBDO) methods and a multitude of these methods have been developed in the past. Among them, the reliability index approach (RIA) [6-13] and the performance measure approach (PMA) [14-18] have been one of the more popular methods. These methods are not perfect and continuous efforts to improve them are being made.
One improvement in the RIA due to its problems on convergence [14] has been implemented by Lin et al. [19] and was called the modified reliability index approach (MRIA). They have determined a new reliability index and the resulting MRIA can evaluate the failure probability correctly, thus solving the convergence problems sometimes encountered in RIA. PMA implements an inverse reliability analysis in determining the performance measures of a design. These obtained performance measures were then used to formulate the probabilistic constraints. PMA has been preferred over MRIA to solve RBDO problems because the inverse reliability analysis was generally more efficient compared to MRIA. Several incidences of numerical instability were encountered in using PMA and will be reported in the latter parts of this paper.

In implementing an RBDO, stability and efficiency are of key concern. In this paper, a hybrid reliability approach (HRA) [20] is presented to achieve stability and efficiency 
using a limited and definite number of iterations. The number of iterations was limited because some designs should be validated by destructive tests or other performance measures [21-24], which can only be done in a limited amount of times due to resource restrictions.

The HRA aims to use the most efficient method for a problem by first determining a selection factor which would determine whether PMA or MRIA would be used. The conditions on which approach would be more appropriate are presented in detail in the later parts of this paper. The approach is then tested on 3 benchmark problems obtained from different literatures $[15,16,18,19,25-30]$ with all approaches used to prove validity of the claim. Strengths or weaknesses of each approach are discussed in detail for each result. The HRA has been previously presented [20] and this paper is differentiated with the elaboration of the selection factor. The results of this paper have also proven an increase in the performance of the HRA. It is claimed that the said approach could be used for safety or design problems with determined constraint functions. Determination of these constraint functions, however, would not be part of the scope of this paper.

\section{Reliability-Based Design Optimization (RBDO)}

The concept of incorporating uncertainty early on in the design stages has paved way to carry out design optimization with the addition of probabilistic reliability as one of the design constraints. The objective is to minimize a cost function or maximize a goal function depending on the nature of the objective while incorporating and satisfying uncertainties or variations. Several methods and approaches have been developed to provide the optimized design in which probabilities of system failures could be reduced to an acceptable level of reliability or safety. A general RBDO can be formulated in the following manner:

$$
\begin{array}{ll}
\underset{\mathbf{d}}{\operatorname{Min}} & y(\mathbf{d}) \\
\text { s.t. } & P\left[g_{i}(\mathbf{X})>0\right] \leq P_{f, i} \quad i=1, \ldots, n,
\end{array}
$$

where $g_{i}(\mathbf{X})$ is the $i$ th performance constraint function of the random variable $\mathbf{X} ; y(\mathbf{d})$ is the objective function of the expected design variable $\mathbf{d}$ of $\mathbf{X}$; and $P_{f, i}$ is the $i$ th allowable probability of failure. In (1), it is computationally costly to evaluate the failure probabilities using the integral of the joint probability density function (JPDF) within the entire infeasible domain, which is expressed as

$$
P[g(\mathbf{X})>0]=\int_{g>0} f(\mathbf{x}) d \mathbf{x},
$$

where $f(\mathbf{x})$ is the JPDF of $\mathbf{X}$. Instead of evaluating this integral, RBDO algorithms have been developed to complete this task.
2.1. Reliability Index Approach (RIA). It was reported that the RIA has convergence problems when the design points fall within the infeasible design domains $[14,19]$. The HasoferLind reliability index [31] as defined in (3) does not include evaluations for infeasible design points and thus produces incorrect evaluations of the failure probabilities for the infeasible design points. Consider

$$
\beta_{\mathrm{HL}, i}=\left\|\mathbf{u}_{i}^{*}\right\|
$$

In (3), $\mathbf{u}_{i}^{*}$ is the most probable failure point (MPFP) for the reliability analysis of $i$ th performance constraint and it is determined by solving the following subproblem:

$$
\begin{array}{ll}
\text { Min } & \left\|\mathbf{u}_{i}\right\| \\
\text { s.t. } & g_{i}\left(\mathbf{u}_{i}\right)=0 .
\end{array}
$$

Figure 1(a) is added to visually illustrate, in three dimensions, the process of finding the MPFP in the $\mathbf{u}_{i}$-space. The nonlinear surface represents the function of $g_{i}\left(\mathbf{u}_{i}\right)$; a color gradient is used to represent function values from $g_{i}\left(\mathbf{u}_{i}\right)>0$ to $g_{i}\left(\mathbf{u}_{i}\right)<0$; the solid boundary is the limit state of $g_{i}\left(\mathbf{u}_{i}\right)=$ 0 . The current design point is located at the origin of the $\mathbf{u}_{i}{ }^{-}$ space and the cross in Figure 1(a) represents the projection of design point on the constraint function. The subproblem (4) is solved and the MPFP $\mathbf{u}_{i}^{*}$ is found on the limit state along the direction of $\nabla_{\mathbf{u}_{\mathbf{i}}} g_{i}\left(\mathbf{u}_{i}^{*}\right)$, illustrated in a separate cross-section A-A in Figure $1(\mathrm{~b})$.

Lin et al. [19] introduced a modified reliability index in (5), which is able to evaluate the failure probabilities correctly regardless of the location of the design points. Consider

$$
\beta_{M, i}=\mathbf{u}_{i}^{*} \cdot \nabla_{\mathbf{u}_{\mathbf{i}}} g_{i}\left(\mathbf{u}_{i}^{*}\right)\left\|\nabla_{\mathbf{u}} g\left(\mathbf{u}_{i}^{*}\right)\right\|^{-1}
$$

The probability of failure is then evaluated using the standard normal cumulative distribution function (CDF) given by (6). Consider

$$
P\left[g_{i}(\mathbf{X})>0\right] \cong \Phi\left(-\beta_{M, i}\right)
$$

Using the inverse operation of $\Phi$ and substituting (6) into (1), (1) is reformulated as

$$
\begin{array}{ll}
\underset{\mathbf{d}}{\operatorname{Min}} & y(\mathbf{d}) \\
\text { s.t. } & -\beta_{M, i}(\mathbf{d}) \leq-\beta_{f, i} \quad i=1, \ldots, n .
\end{array}
$$

For the $i$ th constraint, the modified reliability index is obtained from a first order Taylor expansion of (5) and is expressed as

$$
\begin{aligned}
\beta_{M, i}(\mathbf{d})= & \mathbf{u}_{i}^{*} \cdot \nabla_{\mathbf{u}_{\mathbf{i}}} g_{i}\left(\mathbf{u}_{i}^{*}\right)\left\|\nabla_{\mathbf{u}_{\mathbf{i}}} g_{i}\left(\mathbf{u}_{i}^{*}\right)\right\|^{-1} \\
& -\left(\mathbf{d}-\mathbf{d}^{(k)}\right) \cdot \nabla_{\mathbf{d}} g_{i}\left(\mathbf{u}_{i}^{*}\right)\left\|\nabla_{\mathbf{u}_{\mathbf{i}}} g\left(\mathbf{u}_{i}^{*}\right)\right\|^{-1}
\end{aligned}
$$




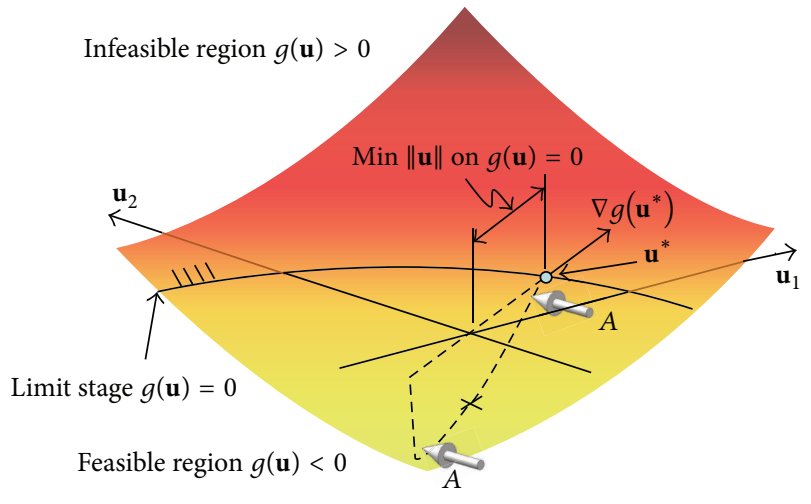

(a)

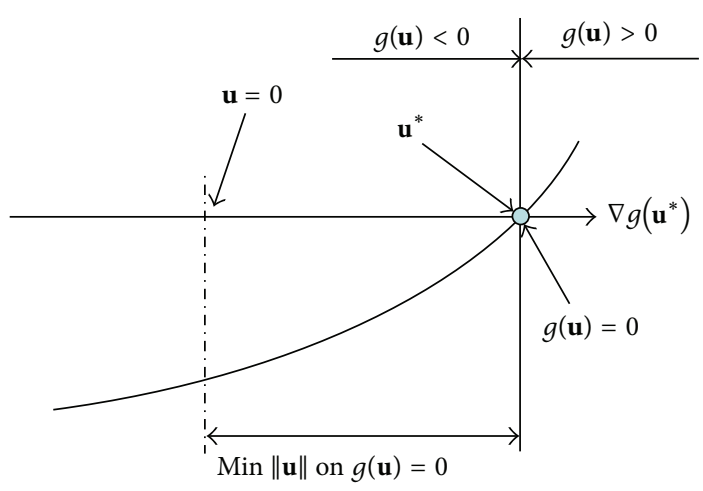

(b)

FIGURE 1: (a) MPFP in standard normal space; (b) cross-sectional view of A-A.

and thus is the modification proposed by Lin et al. [19] for the reliability index in their method. This method is capable of finding the correct optimal solutions within a range of acceptable probabilities of failure. The efficiency of solving the benchmark MPFP problem is the bottleneck of MRIA.

2.2. Performance Measure Approach (PMA). The performance measure approach (PMA) uses an inverse reliability analysis [32] to formulate the probabilistic constraint. An evaluation of a performance measure [14] is first conducted by evaluating (9). Consider

$$
\gamma(\mathbf{d})=F_{g_{i}}^{-1}\left[1-\Phi\left(-\beta_{f, i}\right)\right]
$$

where $F_{g_{i}}(\gamma)$ denotes the probability of $g_{i}(\mathbf{X}) \leq \gamma$. After the performance measure is defined, a general RBDO problem could be formulated as

$$
\begin{array}{ll}
\underset{\mathbf{d}}{\operatorname{Min}} & y(\mathbf{d}) \\
\text { s.t. } & \gamma_{i}(\mathbf{d}) \leq 0 \quad i=1, \ldots, n .
\end{array}
$$

The most probable target point (MPTP) $\mathbf{u}_{i}^{\#}$ is then used to evaluate (10) using the performance measures as the constraints. The subproblem in (11) is formulated to find the optimal solution and the MPTP is determined under the condition of $\beta_{i}>0$. Consider

$$
\begin{array}{ll}
\operatorname{Max} & g_{i}\left(\mathbf{u}_{i}\right) \\
\text { s.t. } & \left\|\mathbf{u}_{i}\right\|=\beta_{f, i} .
\end{array}
$$

The MPTP is determined from the distance of $\beta_{f, i}$ away from the design point along the direction of $\nabla_{\mathbf{u}} g_{i}\left(\mathbf{u}_{i}^{\#}\right)$. Subproblem (11) considers the inverse reliability analysis and Figure 2(a) illustrates the process of finding the MPTP in the standard normal space. The red $\beta_{f, i}$-contour represents the area of the allowable probability surrounding the current design and the blue contour is the projection of the $\beta_{f, i}$-contour on the surface of $g_{i}\left(\mathbf{u}_{i}\right)$. The B-B cross-section in Figure 2(b) shows how the location of the MPTP is determined at the maximum function value on the $\beta_{f, i}$-geometry along the direction of $\nabla_{\mathbf{u}} g_{i}\left(\mathbf{u}_{i}^{\#}\right)$.

The performance measure for the $i$ th constraint for problem (9) is then approximated as

$$
\gamma_{i}(\mathbf{d})=g_{i}\left(\mathbf{u}_{i}^{\#}\right)+\left(\mathbf{d}-\mathbf{d}^{(k)}\right) \cdot \nabla_{\mathbf{d}} g_{i}\left(\mathbf{u}_{i}^{\#}\right) .
$$

Some researchers $[17,33,34]$ preferred to use PMA for RBDO problems due to its efficiency for their problem by utilizing mean-value iterative methods, such as a hybrid mean value (HMV) method [17, 35].

2.3. Instability of PMA. The subproblem as expressed in (11) does not guarantee finding the most probable target that satisfies $g_{i}\left(\mathbf{u}_{i}\right)=0$ for all possible locations. Some researchers [18] have found ways to ensure that the locations would not fall within the improbable location but there might be some scenarios where constraints are constantly changing or are unknown thus selecting a design point falling within the improbable areas is still a possibility. The additional function calls from the optimization may be too costly in complicated engineering problems and other methods require additional function evaluations. In Figure 3, an attempt is made to visually illustrate this phenomenon through several different probable cases.

In Figures 3 and 4 different possible scenarios are explored in one plane, which may represent different design points. Design points $\mathbf{d}_{1}$ and $\mathbf{d}_{2}$ fall within the feasible region. For $\mathbf{d}_{1}$, the most probable target of zero function value of $g$ is determined at the maximum function measure on the $\beta_{f}$-contour, which defines the MPTP $\mathbf{d}_{1}^{\#}$. The performance measure is then evaluated by mapping $\mathbf{d}_{1}^{\#}$ to $\mathbf{u}$-space and substituting $\mathbf{u}_{1}^{\#}$ to (9). For $\mathbf{d}_{2}$, the $\beta_{f}$-contour is in contact with the limit state and the MPTP is found exactly at 


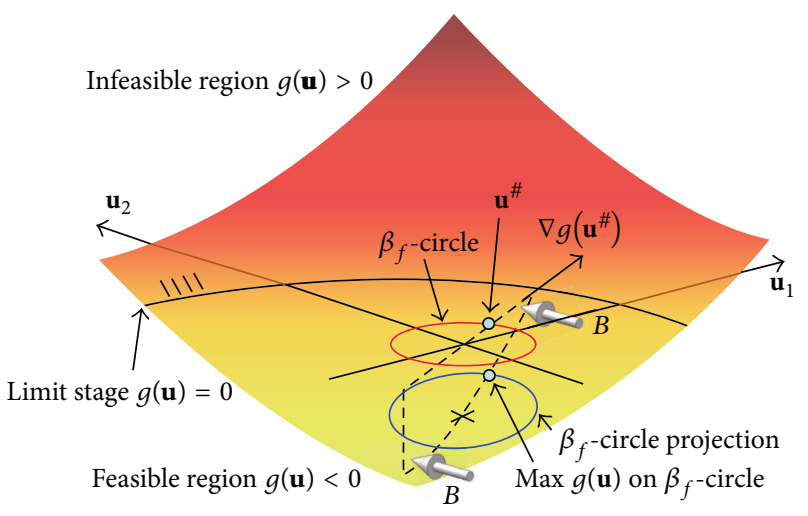

(a)

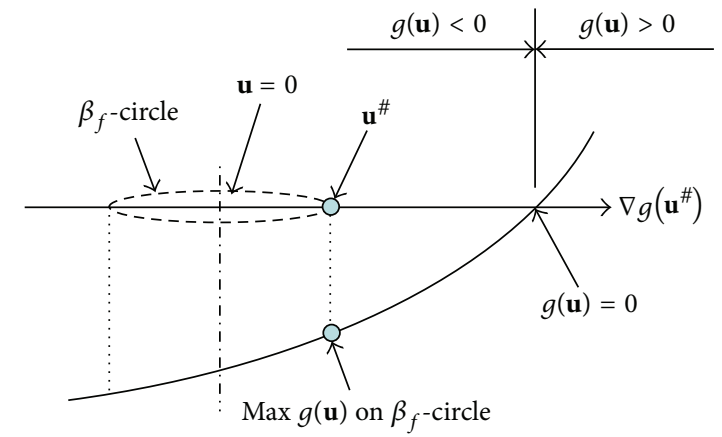

(b)

FIGURE 2: (a) MPTP in standard normal space; (b) cross-sectional view of B-B.

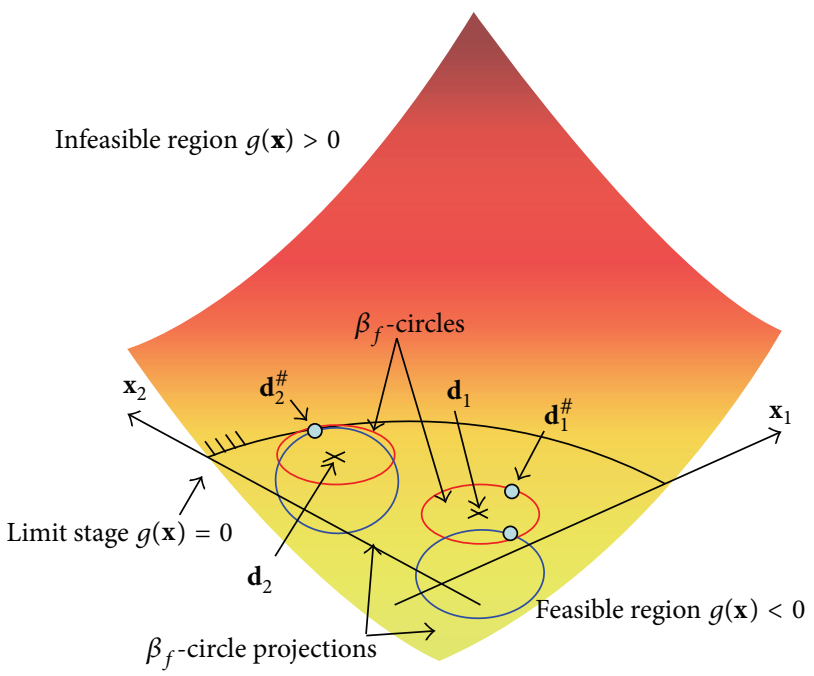

(a)

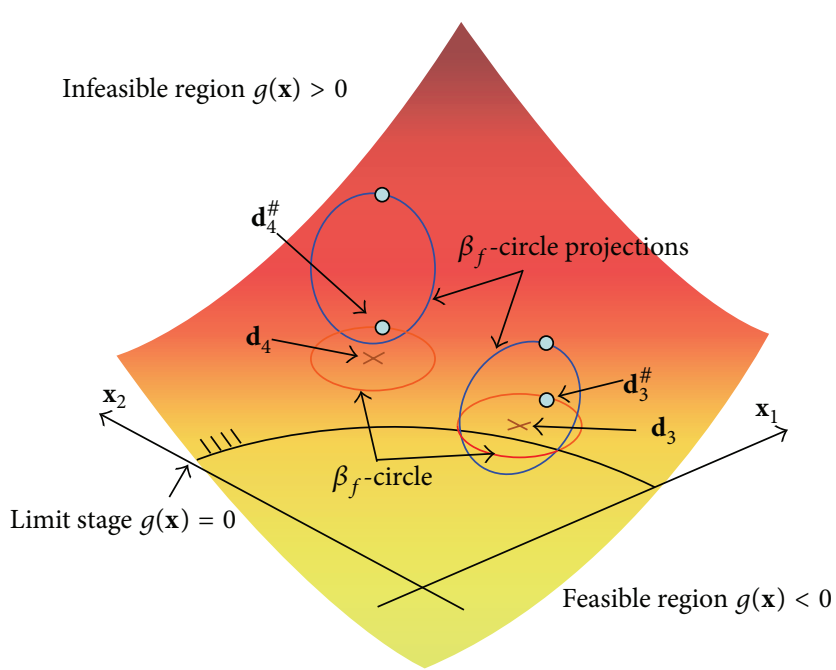

(b)

FIGURE 3: Generalization of different design points: (a) point $\mathbf{d}_{1}$ in the feasible region and point $\mathbf{d}_{2}$ with its MPTP on the limit state; (b) point $\mathbf{d}_{3}$ with its $\beta_{f}$-contour crossing the limit state and point $\mathbf{d}_{4}$ in the infeasible region.

the intersection of the $\beta_{f}$-contour and the limit state. In this condition, the MPTP is determined at $g_{i}\left(\mathbf{u}_{i}^{\#}\right)=0$ on the $\beta_{f^{-}}$ contour.

The next two points, $\mathbf{d}_{3}$ and $\mathbf{d}_{4}$, are cases in which the design points fall outside the feasible regions. The $\beta_{f}$-contour for the design point $\mathbf{d}_{3}$ crosses the limit state and the targets of zero function values can be found at the intersection of the $\beta_{f}$-contour and limit state. However, the MPTP will be determined in the infeasible region instead of on the limit state using the subproblem in (11) but it is not the most probable target of $g(\mathbf{x})=0$. Lastly, for point $\mathbf{d}_{4}$, the MPTP is found away from the limit state on the $\beta_{f}$-contour and it produces inappropriate inverse reliability analysis. These cases were encountered in solving the benchmark problems using the PMA and will be presented later in the numerical examples.

\section{A Multifaceted Approach for RBDO}

3.1. Investigations of Active Probabilistic Constraints of MRIA and PMA. Lin et al. [20] proposed the HRA and expected to use the methods of MRIA and PMA in cases where they perform the best. MRIA is able to find the optimal solution in any design location but the PMA, on the other hand, is able to solve the RBDO problem using the inverse reliability analysis very efficiently.

MRIA and PMA optimality conditions are first presented to demonstrate that both approaches obtain the same point in the RBDO problem, given the same probabilistic constraints. This is necessary since even though each approach uses a different perspective, they are trying to obtain the same optimal solution under constraints. Thus, both approaches can be used interchangeably whenever their performance is superior. 


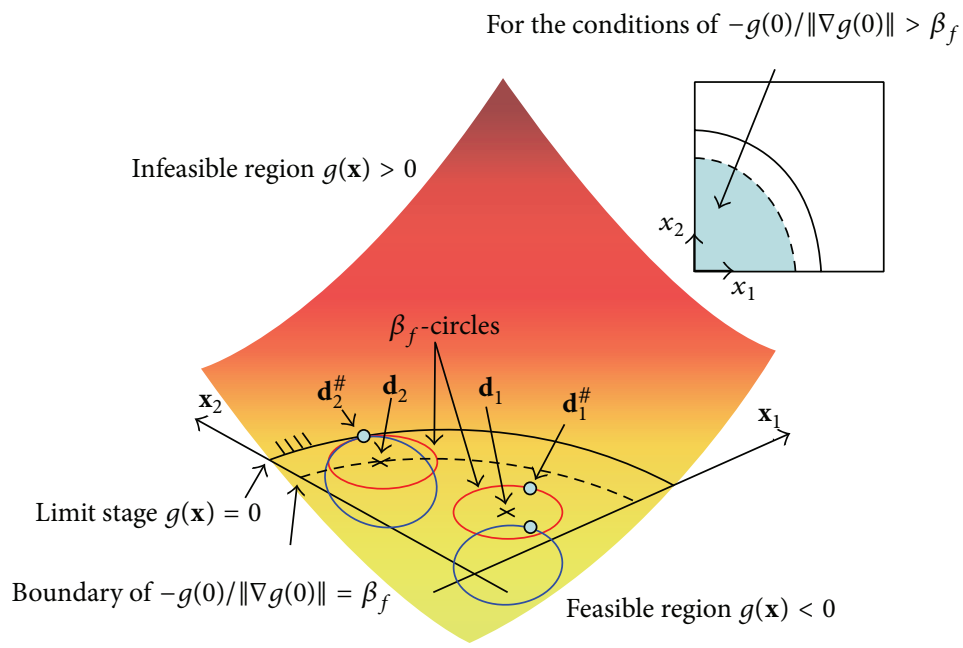

(a)

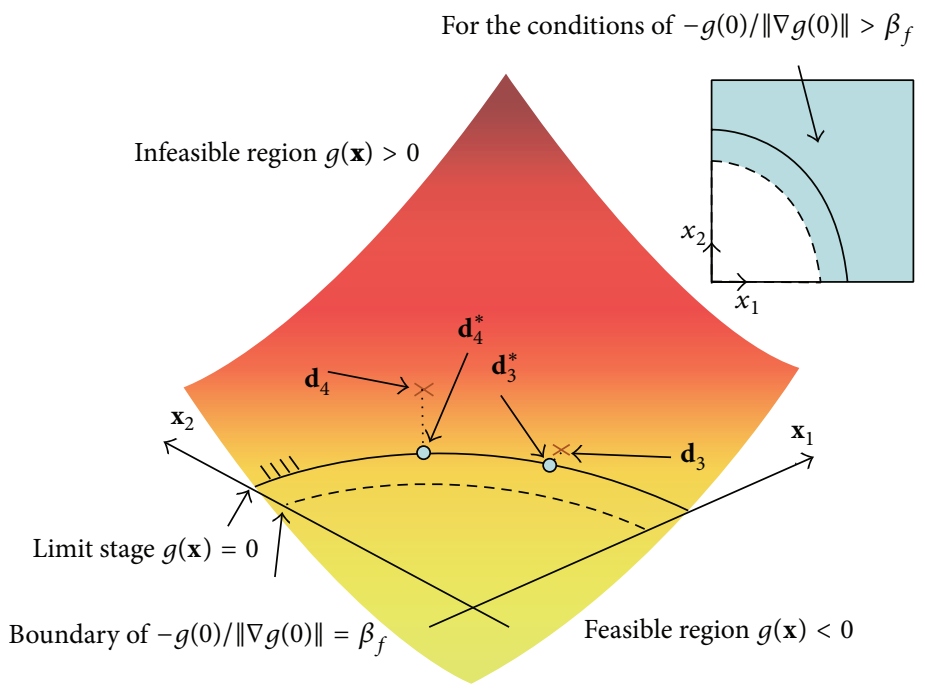

(b)

FIGURE 4: Selection of algorithms based on the relative relationship between Cornell reliability index and $\beta_{f}$ : (a) conditions where PMA is used; (b) conditions when MRIA is used.

The optimality conditions of both methods are first investigated to identify their similarity and differences. A selection factor is derived according to the relations between the two methods and utilized to determine whether they would be considered during the optimization processes of RBDO.

It is given that the active probabilistic constraints using both MRIA and PMA are the same. In other words, both methods should be able to generate the same approximations of probabilistic constraints. They are numerically shown in (13) and (14), respectively, when the design variables are close to the optimal solution of RBDO problem in (1). Consider

$$
\begin{aligned}
-\mathbf{u}_{i}^{*} & \cdot \nabla_{\mathbf{u}_{\mathbf{i}}} g_{i}\left(\mathbf{u}_{i}^{*}\right)\left\|\nabla_{\mathbf{u}_{\mathbf{i}}} g_{i}\left(\mathbf{u}_{i}^{*}\right)\right\|^{-1}+\beta_{f, i} \\
& +\left(\mathbf{d}-\mathbf{d}^{(k)}\right) \cdot \nabla_{\mathbf{d}} g_{i}\left(\mathbf{u}_{i}^{*}\right)\left\|\nabla_{\mathbf{u}_{\mathbf{i}}} g\left(\mathbf{u}_{i}^{*}\right)\right\|^{-1}=0,
\end{aligned}
$$

$$
\begin{array}{r}
g_{i}\left(\mathbf{u}_{i}^{\#}\right)\left\|\nabla_{\mathbf{u}_{\mathbf{i}}} g\left(\mathbf{u}_{i}^{\#}\right)\right\|^{-1}+\left(\mathbf{d}-\mathbf{d}^{(k)}\right) \\
\cdot \nabla_{\mathbf{d}} g_{i}\left(\mathbf{u}_{i}^{\#}\right)\left\|\nabla_{\mathbf{u}_{\mathbf{i}}} g\left(\mathbf{u}_{i}^{\#}\right)\right\|^{-1}=0 .
\end{array}
$$

Equations (13) and (14) were derived from the active conditions of the inequality constraints in (7) and (10), respectively. At the optimal solution, $\nabla_{\mathbf{d}} g_{i}\left(\mathbf{u}_{i}^{*}\right) \cong \nabla_{\mathbf{d}} g_{i}\left(\mathbf{u}_{i}^{\#}\right)$ and the following relation could be obtained by substituting this condition and subtracting (13) from (14). Consider

$$
\begin{aligned}
g_{i}\left(\mathbf{u}_{i}^{\#}\right)\left\|\nabla_{\mathbf{u}_{\mathbf{i}}} g\left(\mathbf{u}_{i}^{\#}\right)\right\|^{-1}+\mathbf{u}_{i}^{*} \\
\cdot \nabla_{\mathbf{u}_{\mathbf{i}}} g_{i}\left(\mathbf{u}_{i}^{*}\right)\left\|\nabla_{\mathbf{u}_{\mathbf{i}}} g_{i}\left(\mathbf{u}_{i}^{*}\right)\right\|^{-1}-\beta_{f, i}=0 .
\end{aligned}
$$

3.2. Derivation of Selection Factor Using the Generalized Formulations of RBDO Algorithms. Equation (15) can also 
be derived from the difference of the unified reliability formulations (URFs) [36] of MRIA and PMA. Lin [36] has found the generalized mathematical expression of the linearly approximated probabilistic constraints of various RBDO algorithms. The URFs of MRIA and PMA have been derived as (16) and (17), respectively. Consider

$$
\begin{aligned}
& {\left[\mathbf{d}-\mathbf{x}_{i}^{*}+g_{i}\left(\mathbf{x}_{i}^{*}\right) \boldsymbol{\sigma}^{2} \cdot \frac{\nabla_{\mathbf{d}} g_{i}\left(\mathbf{x}_{i}^{*}\right)}{\left\|\boldsymbol{\sigma} \cdot \nabla_{\mathbf{d}} g_{i}\left(\mathbf{x}_{i}^{*}\right)\right\|^{2}}\right.} \\
& \left.+\beta_{f, i} \boldsymbol{\sigma}^{2} \cdot \frac{\nabla_{\mathbf{d}} g_{i}\left(\mathbf{x}_{i}^{*}\right)}{\left\|\boldsymbol{\sigma} \cdot \nabla_{\mathbf{d}} g_{i}\left(\mathbf{x}_{i}^{*}\right)\right\|}\right] \cdot \nabla_{\mathbf{d}} g_{i}\left(\mathbf{x}_{i}^{*}\right) \leq 0, \\
& {\left[\mathbf{d}-\mathbf{x}_{i}^{\#}+g_{i}\left(\mathbf{x}_{i}^{\#}\right) \boldsymbol{\sigma}^{2} \cdot \frac{\nabla_{\mathbf{d}} g_{i}\left(\mathbf{x}_{i}^{\#}\right)}{\left\|\boldsymbol{\sigma} \cdot \nabla_{\mathbf{d}} g_{i}\left(\mathbf{x}_{i}^{\#}\right)\right\|^{2}}\right.} \\
& \left.+\beta_{f, i} \boldsymbol{\sigma}^{2} \cdot \frac{\nabla_{\mathbf{d}} g_{i}\left(\mathbf{x}_{i}^{\#}\right)}{\left\|\boldsymbol{\sigma} \cdot \nabla_{\mathbf{d}} g_{i}\left(\mathbf{x}_{i}^{\#}\right)\right\|}\right] \cdot \nabla_{\mathbf{d}} g_{i}\left(\mathbf{x}_{i}^{\#}\right) \leq 0,
\end{aligned}
$$

where $\mathbf{x}_{i}^{*}$ and $\mathbf{x}_{i}^{\#}$, respectively, represent the MPFP and MPTP in the original design space. When both inequality constraints are active and the gradient vectors evaluated at MPFP and MPTP are the same, the following relation is obtained:

$$
\begin{aligned}
-\mathbf{x}_{i}^{*} & +g_{i}\left(\mathbf{x}_{i}^{*}\right) \boldsymbol{\sigma}^{2} \cdot \frac{\nabla_{\mathbf{d}} g_{i}\left(\mathbf{x}_{i}^{*}\right)}{\left\|\boldsymbol{\sigma} \cdot \nabla_{\mathbf{d}} g_{i}\left(\mathbf{x}_{i}^{*}\right)\right\|^{2}} \\
& +\beta_{f, i} \boldsymbol{\sigma}^{2} \cdot \frac{\nabla_{\mathbf{d}} g_{i}\left(\mathbf{x}_{i}^{*}\right)}{\left\|\boldsymbol{\sigma} \cdot \nabla_{\mathbf{d}} g_{i}\left(\mathbf{x}_{i}^{*}\right)\right\|} \\
= & -\mathbf{x}_{i}^{\#}+g_{i}\left(\mathbf{x}_{i}^{\#}\right) \boldsymbol{\sigma}^{2} \cdot \frac{\nabla_{\mathbf{d}} g_{i}\left(\mathbf{x}_{i}^{\#}\right)}{\left\|\boldsymbol{\sigma} \cdot \nabla_{\mathbf{d}} g_{i}\left(\mathbf{x}_{i}^{\#}\right)\right\|^{2}} \\
& +\beta_{f, i} \boldsymbol{\sigma}^{2} \cdot \frac{\nabla_{\mathbf{d}} g_{i}\left(\mathbf{x}_{i}^{\#}\right)}{\left\|\boldsymbol{\sigma} \cdot \nabla_{\mathbf{d}} g_{i}\left(\mathbf{x}_{i}^{\#}\right)\right\|} .
\end{aligned}
$$

Since $g_{i}\left(\mathbf{x}_{i}^{*}\right)=0$ and $\mathbf{x}_{i}^{\#}-\mathbf{d}^{(k)}=\beta_{f, i} \boldsymbol{\sigma}^{2}$. $\nabla_{\mathbf{d}} g_{i}\left(\mathbf{x}_{i}^{\#}\right)\left\|\boldsymbol{\sigma}^{2} \cdot \nabla_{\mathbf{d}} g_{i}\left(\mathbf{x}_{i}^{\#}\right)\right\|^{-1},(18)$ is rewritten as

$$
\begin{aligned}
-\mathbf{x}_{i}^{*}+\beta_{f, i} \boldsymbol{\sigma}^{2} \cdot \frac{\nabla_{\mathbf{d}} g_{i}\left(\mathbf{x}_{i}^{*}\right)}{\left\|\boldsymbol{\sigma} \cdot \nabla_{\mathbf{d}} g_{i}\left(\mathbf{x}_{i}^{*}\right)\right\|} \\
=-\mathbf{d}^{(k)}+g_{i}\left(\mathbf{x}_{i}^{\#}\right) \boldsymbol{\sigma}^{2} \cdot \frac{\nabla_{\mathbf{d}} g_{i}\left(\mathbf{x}_{i}^{\#}\right)}{\left\|\boldsymbol{\sigma} \cdot \nabla_{\mathbf{d}} g_{i}\left(\mathbf{x}_{i}^{\#}\right)\right\|^{2}} .
\end{aligned}
$$

A dot product between (19) and $\boldsymbol{\sigma} \cdot \nabla_{\mathbf{d}} g_{i}\left(\mathbf{x}_{i}^{*}\right)$ produces

$$
\begin{gathered}
-\mathbf{u}_{i}^{*} \cdot \boldsymbol{\sigma} \cdot \nabla_{\mathbf{d}} g_{i}\left(\mathbf{x}_{i}^{*}\right)+\beta_{f, i}\left\|\boldsymbol{\sigma} \cdot \nabla_{\mathbf{d}} g_{i}\left(\mathbf{x}_{i}^{*}\right)\right\| \\
=g_{i}\left(\mathbf{x}_{i}^{\#}\right) \frac{\nabla_{\mathbf{d}} g_{i}\left(\mathbf{x}_{i}^{\#}\right) \cdot \boldsymbol{\sigma}^{2} \cdot \nabla_{\mathbf{d}} g_{i}\left(\mathbf{x}_{i}^{*}\right)}{\left\|\boldsymbol{\sigma} \cdot \nabla_{\mathbf{d}} g_{i}\left(\mathbf{x}_{i}^{\#}\right)\right\|^{2}} .
\end{gathered}
$$

The relation of $\nabla_{\mathbf{d}} g_{i}\left(\mathbf{x}_{i}^{\#}\right) \cong \nabla_{\mathbf{d}} g_{i}\left(\mathbf{x}_{i}^{*}\right)$ simplifies (20) to the following final form:

$$
\mathbf{u}_{i}^{*} \cdot \frac{\nabla_{\mathbf{u}} g_{i}\left(\mathbf{x}_{i}^{*}\right)}{\left\|\nabla_{\mathbf{u}} g_{i}\left(\mathbf{x}_{i}^{*}\right)\right\|}=\beta_{f, i}-\frac{g_{i}\left(\mathbf{x}_{i}^{*}\right)}{\left\|\nabla_{\mathbf{u}} g_{i}\left(\mathbf{x}_{i}^{*}\right)\right\|}
$$

which confirms derived Equation (15).

Lin et al. [20] defined a selection factor $S_{i}$ based on the ratio of right-hand-side of (21) over the left-hand-side. As the ratio is close to or equal to one, the approximations of MRIA and PMA are very similar. Therefore, it was suggested to utilized the efficient PMA when $\left\|S_{i}-1\right\|<D$, where $D$ is a user-defined threshold parameter. Otherwise, the stable MRIA was utilized to formulate the $i$ th probabilistic constraint. However, the selection of the threshold parameter is crucial for the performance of HRA. In this paper, an improved definition of selection factor is proposed for the multifaceted selection in HRA.

3.3. Improvements in the Selection Factor for Hybrid Reliability Approach. In this paper, a conditional selection factor is proposed to determine whether MRIA and PMA should be utilized during the optimization processes without the decision of threshold parameter $D$. In order to enhance the numerical performance of HRA, the mean-value approximation is utilized to reformulate (15) as the following equation:

$$
\begin{aligned}
& {\left[g_{i}(0)+\mathbf{u}_{i}^{\#} \cdot \nabla_{\mathbf{u}_{\mathbf{i}}} g(0)\right]\left\|\nabla_{\mathbf{u}_{\mathbf{i}}} g(0)\right\|^{-1}} \\
& \quad+\mathbf{u}_{i}^{*} \cdot \nabla_{\mathbf{u}_{\mathbf{i}}} g_{i}(0)\left\|\nabla_{\mathbf{u}_{\mathbf{i}}} g(0)\right\|^{-1}-\beta_{f, i}=0 .
\end{aligned}
$$

Furthermore, the mean-value approximations of MPFP and MPTP near the optimal solution are given by $\mathbf{u}_{i}^{*} \cong \mathbf{u}_{i}^{\#} \cong$ $\beta_{f, i} \nabla_{\mathbf{u}_{\mathbf{i}}} g_{i}(0)\left\|\nabla_{\mathbf{u}_{\mathbf{i}}} g(0)\right\|^{-1}$. A new selection factor $S_{i}$ is then derived as

$$
S_{i} \equiv g_{i}(0)\left\|\nabla_{\mathbf{u}_{\mathbf{i}}} g(0)\right\|^{-1}+\beta_{f, i} .
$$

After evaluating (23), three cases can then be generalized to describe $S_{i}$. These three cases are elaborated below and define the selection between PMA and MRIA.

(i) Case 1 is when $S_{i}=0$, the Cornell reliability index [37] $\beta_{C, i} \equiv-g_{i}(0)\left\|\nabla_{\mathbf{u}_{\mathbf{i}}} g(0)\right\|^{-1}$ under mean-value firstorder second-moment (MVFOSM) [38] is the desired reliability index, $\beta_{f, i}$, and the failure probability from the MRIA and the PMA is approximately the same. In this case, the PMA would be chosen to perform an efficient inverse reliability analysis.

(ii) Case 2 is when $S_{i}<0$ and the Cornel reliability index is larger than $\beta_{f, i}$ indicating that the beta-contour is located within the feasible region, the efficient inverse reliability analysis can find the MPTP of $g_{i}\left(\mathbf{u}_{i}\right)=0$ and PMA is the logical choice for this case.

(iii) Lastly, Case 3 is when $S_{i}>0$, the $\beta_{f, i}$-contour is either crossing the limit state or entirely located outside the feasible region. To avoid the numerical instability experienced in PMA, MRIA is chosen as the approach to find the optimal conditions. 
These three cases could further be simplified by expressing them into a decision RBDO algorithm with the following rule:

$$
\begin{aligned}
& \text { PMA is used. for } S_{i} \leq 0 \text {; } \\
& \text { otherwise, MRIA is used. }
\end{aligned}
$$

It is novel to use a conditional algorithm to solve a problem using PMA and MRIA in cases where their strengths are used, PMA using an efficient inverse reliability and MRIA utilizing its stability. The evaluation of the selection factor, $S_{i}$, from (23) does not generate additional functional evaluations because both $g_{i}(0)$ and $\nabla_{\mathbf{u}_{i}} g(0)$ are used in performing the analysis in PMA and MRIA. It is expected that the proposed HRA will be more efficient in finding the optimal solution. Figure 4 visually represents the algorithm selection process.

In Figure 4(a), design point $\mathbf{d}_{1}$ is located in the region of $S_{i} \leq 0$. The Cornell reliability index is larger than or maybe equal to the allowable reliability index and PMA is implemented in formulating the probabilistic constraint based on (10) and (12). Design point $\mathbf{d}_{2}$ is a point where the corresponding $\beta_{f, i}$-contour contacts the limit state and it is located on the boundary of $S_{i}=0$ and PMA is used and the MPTP is found on the limit state. Figure 4(b), on the other hand, shows infeasible design points $\mathbf{d}_{3}$ and $\mathbf{d}_{4}$, where $S_{i}>0$ and $\beta_{C, i}<\beta_{f, i}$. In these cases, the MRIA is utilized to produce the probabilistic constraints from (7) and (8) and stable reliability analyses are expected.

The proposed procedure of HRA establishes a multifaceted approach in solving RBDO problems and could be also applied to other methods after carefully knowing the strengths and weaknesses of each method and formulating a selection factor based on the conditions in which these methods perform well. As mentioned in the RIA section of these paper, different approaches such as AMV [39], FORM $[31,40]$, and SORM [38] could be utilized in finding the MPFP. Meanwhile, other PMA methods, such as HMV [35] and HMV+ [17], can also be utilized in obtaining the MPTP. The proposed approach is also adaptive to probabilistic constraints. It can be applied to linear or high-order expansions such as that related to safety and reliability information. Performance-based shifting $[16,41,42]$, variable-based shifting [43], and nonlinear transformations in nonnormally distributed random variables [15] can be covered by the proposed approach.

\section{Numerical Examples}

This section makes a comparative investigation of MRIA, PMA, and HRA using a set of benchmark examples obtained from related studies $[15,16,19,25-29]$. The iteration processes and optimal results performed at randomly selected starting points were investigated with the function evaluations used as efficiency indicators. Any violation in the conditions of the allowable failure probabilities was further investigated using Monte Carlo Simulations. The results of the Monte Carlo Simulations are utilized as an indicator for correctness and stability.
All problems were examined with $20 \times 20$ uniformly distributed starting points from the design domain and the optimization process stops when a relative difference of $10^{-3}$ from the objective function is reached. The maximum iteration for finding the MPFP and the global iteration loop was limited to five [25].

4.1. Application on a Nonlinear Problem I. The first benchmark problem is a two-bar frame problem. The full statement of the problem can be found at [15]. The formulation of the optimization problem for this paper is stated as

$$
\begin{array}{ll}
\underset{\mathbf{d}}{\operatorname{Min}} & y(\mathbf{d})=d_{1}+d_{2} \\
\text { s.t. } & P_{i}\left[g_{i}(\mathbf{X})>0\right] \leq P_{f} \quad i=1, \ldots, 3 \\
& 0 \leq d_{j} \leq 10 \quad j=1,2,
\end{array}
$$

where

$$
\begin{gathered}
g_{1}(\mathbf{X})=1-\frac{X_{1}^{2} X_{2}}{20}, \\
g_{2}(\mathbf{X})=1-\frac{\left(X_{1}+X_{2}-5\right)^{2}}{30}-\frac{\left(X_{1}+X_{2}-12\right)^{2}}{120}, \\
g_{3}(\mathbf{X})=1-\frac{80}{X_{1}^{2}+8 X_{2}+5}
\end{gathered}
$$

with the probabilistic constraint of $P_{f}=0.13 \%$ to follow $3 \sigma$ criterion. The design point $\mathbf{X}$ has the standard deviation of $\left[\sigma_{1}, \sigma_{2}\right]=[0.3,0.3]$ and is a mutually independent, normally distributed random variable. The problem and the optimal solution to the problem are graphically represented in Figure 5.

The required function evaluations (FE) to solve the RBDO problem using 400 starting points are demonstrated in Figure 6. The location of the bars represents the decisions of the starting points. The heights of the bars record the number of FE for the optimization process with the corresponding starting points.

The design points may fall into the infeasible regions during the optimization process to determine whether the starting points are feasible or not. At first glance, Figure 6(a) shows that MRIA has some points with high FEs, most of which are outside the feasible region. Figure 6(b), on the other hand, shows a more stable distribution in the FEs. It is quite noticeable that the HRA in Figure 6(c) has lowest FE since it adopted the best solution of both approaches. The average of the FE among all possible starting points is taken as the efficiency indicator, later tabulated in Table 1.

In evaluating the correctness of the optimal solutions, Monte Carlo Simulations (MCS) was used to study the probability of failure corresponding to each starting point. The sum of violations (SOV) of the allowable probability is calculated by $\sum_{i=1}^{n}\left(P_{\mathrm{MCS}, i}-P_{f, i}\right)$, where $P_{\mathrm{MCS}, i}$ is used to denote the probability of failure of the $i$ th constraint based on the MCS with $10^{6}$ sampling points. Figure 7 shows the SOVs of all three methods. 


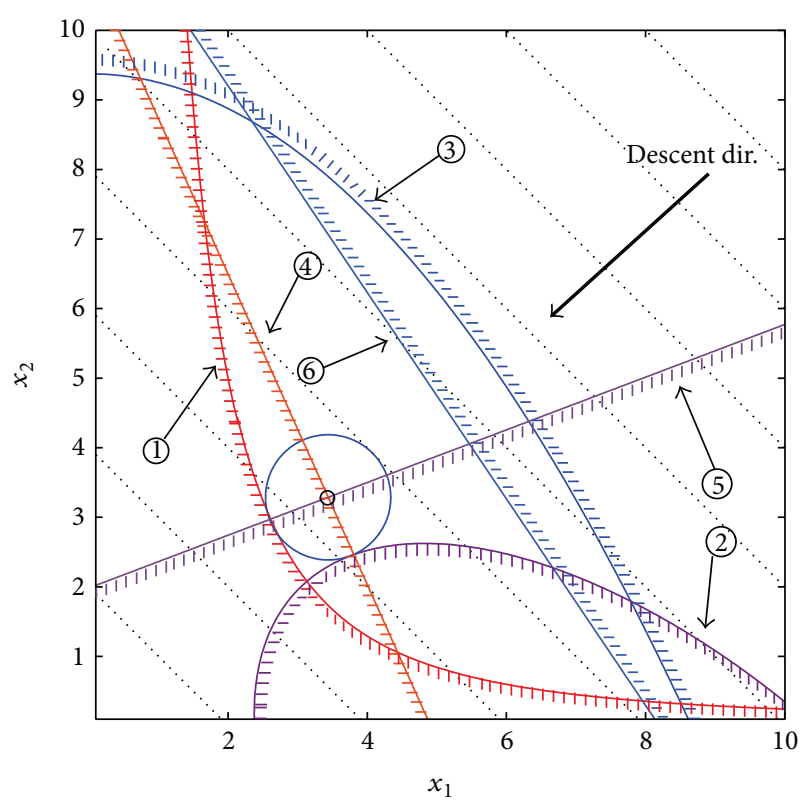

$\begin{array}{ll}\text {.e. Obj. func. } & \text { (5) Prob. constr. \#2 } \\ \text { (1) Deter. constr. \#1 } & \text { (6) Prob. constr. \#3 } \\ \text { (2) Deter. constr. \#2 } & \bigcirc \beta \text {-circle } \\ \text { (3) Deter. constr. \#3 } & 0 \text { Optimal point } \\ \text { (4) Prob. constr. \#1 } & \end{array}$

FIGURE 5: Graphical representation of Example 1 [20].

TABLE 1: FE and SOV of 400 starting points for Example 1.

\begin{tabular}{lccc}
\hline Approach & Average FE & Average SOV & $\begin{array}{c}\text { Number of } \\
\text { points with } \\
\text { high SOV }\end{array}$ \\
\hline MRIA & 282.9 & $0.018 \%$ & 0 \\
PMA & 274.5 & $52.199 \%$ & 158 \\
HRA & 256.2 & $0.018 \%$ & 0 \\
\hline
\end{tabular}

${ }^{*}$ Sum of violations $>1 \%$.

Figure 7(a) demonstrates that MRIA has a very low SOV proving that it can find the correct optimal solution within the allowable probability of failure in any given starting point. This property was adopted by HRA as seen in Figure 7(c). Figure 7(b), on the other hand, shows the instability of PMA on points outside the feasible regions. The results of the FEs and SOVs are summarized on Table 1.

The first glance claims are validated with the summary and it is notable that HRA has the lowest number of FEs, taking good results from the PMA and MRIA. The SOV of MRIA is almost close to zero and was adopted by the HRA. PMA on the other hand had a large number of violations and 158 stating points produce SOVs higher than $1 \%$, meaning that in 158 points, approximations of the PMA are incorrect. The locations of the 158 incorrect points for PMA are shown in Figure 8.
TABLE 2: FE and SOV of 400 starting points for Example 2.

\begin{tabular}{lccc}
\hline Approach & Average FE & Average SOV & $\begin{array}{c}\text { Number of } \\
\text { points with } \\
\text { high SOV }\end{array}$ \\
\hline MRIA & 204.9 & $0.024 \%$ & 0 \\
PMA & 155.9 & $0.023 \%$ & 0 \\
HRA & 167.0 & $0.024 \%$ & 0 \\
\hline
\end{tabular}

${ }^{*}$ Sum of violations $>1 \%$.

The Cornell reliability index in some instances of the 158 starting points, presented above, as a value lower than that of the allowable reliability index and the MPTP in (11), cannot guarantee to get the most probable target toward the limit state. In such cases, the HRA follows (4) and uses the MRIA to solve the RBDO analysis up to such time that the design point is led into conditions that $S_{i} \leq 0$ and the efficient inverse reliability analysis of PMA is used. This ensures that HRA keeps the stability of MRIA and maintains the efficiency of PMA.

4.2. Application on a Nonlinear Problem II. A second nonlinear benchmark problem [30], which considers uncertainties in the design variables in a real safety system, is presented as follows:

$$
\begin{array}{ll}
\underset{\mathbf{d}}{\operatorname{Min}} & y(\mathbf{d})=4 d_{1}^{2}+d_{1}-d_{2}-2.5 \\
\text { s.t. } & P_{i}\left[g_{i}(\mathbf{X})>0\right] \leq 0.13 \% \quad i=1, \ldots, 2 \\
& d_{1} \in[-1,2], \quad d_{2} \in[0.1,3] \\
& {\left[\sigma_{1}, \sigma_{2}\right]=[0.1,0.1],}
\end{array}
$$

where

$$
\begin{aligned}
& g_{1}(\mathbf{X})=-X_{2}^{2}+1.5 X_{1}^{2}-2 X_{1}+1 \\
& g_{1}(\mathbf{X})=X_{2}^{2}+2 X_{1}^{2}-2 X_{1}-4.25
\end{aligned}
$$

The problem and the optimal solution to the problem are graphically presented in Figure 9. In this illustration, the descent direction arrows were used to simplify the visualization of the location of the optimal point. There were no stability incidents reported in this given problem in the utilization of all the approaches. The performances of each approach are summarized in Table 2.

When no numerical instabilities were present, the PMA, which uses the lowest function evaluations averagely, seems to be the most efficient. The HRA had higher average FEs compared to PMA. This is due to some cases in which $S_{i}>0$ and HRA had to use MRIA. This example shows the cost of computation using MRIA. For this example, the average FEs are around $30 \%$ compared to PMA. The HRA on the other hand suffers an increase of approximately $7 \%$ in FEs compared to PMA due to the cases in which $S_{i}>0$. The MRIA has stable optimization processes but the MPFP-searching processes are costly. 


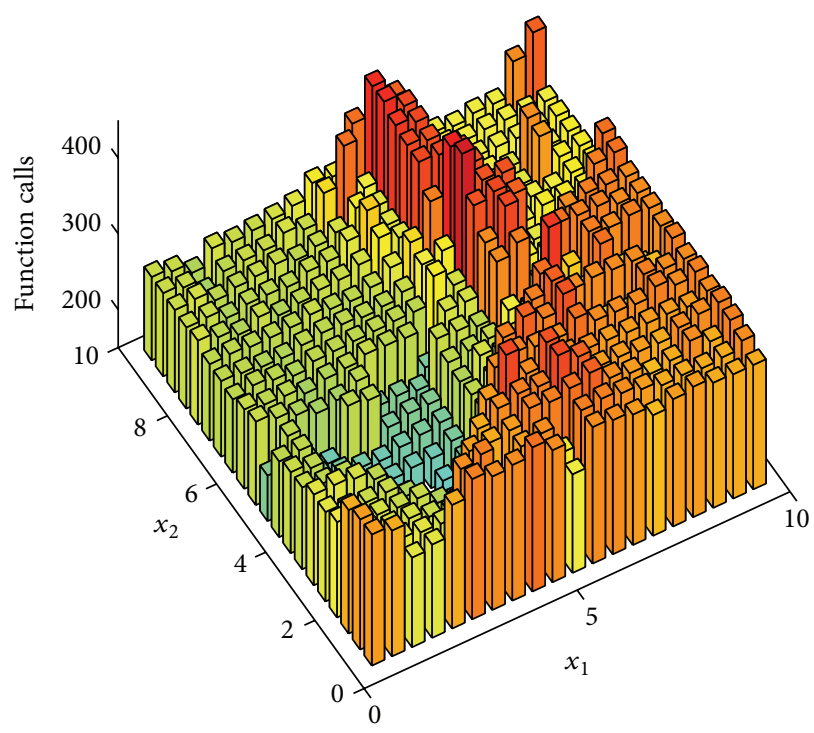

(a)

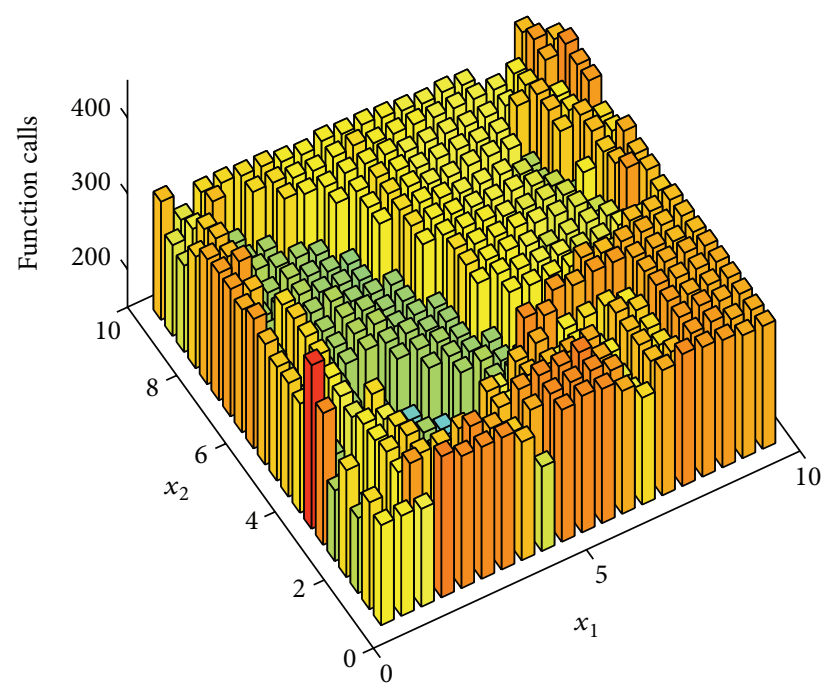

(b)

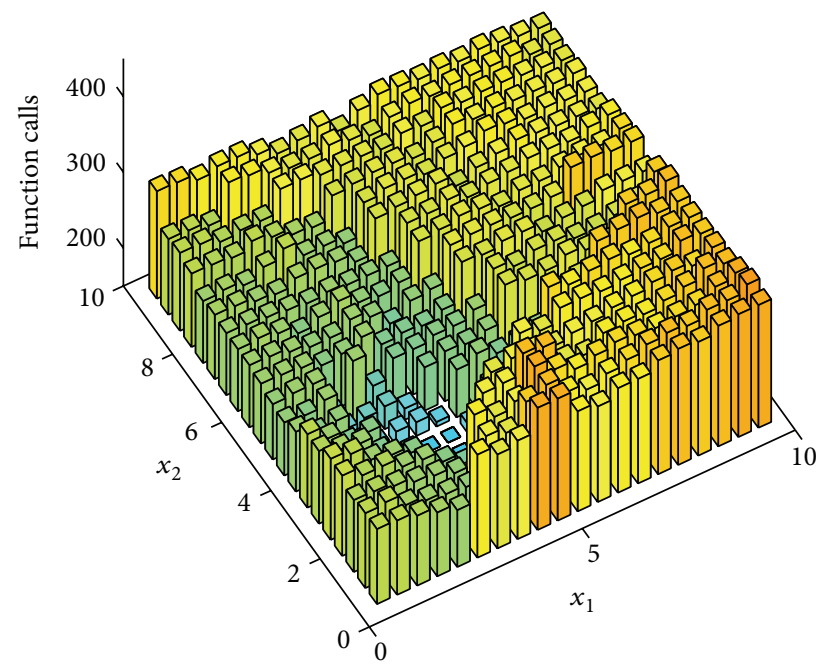

(c)

FIGURE 6: Function evaluations for 400 different starting points using (a) MRIA, (b) PMA, and (c) HRA.

4.3. Application on a Highly Nonlinear Problem. The third benchmark problem originally proposed in [42] is a highly nonlinear two-dimensional RBDO problem. The optimization problem was presented as

$$
\begin{array}{ll}
\underset{\mathbf{d}}{\operatorname{Min}} & y(\mathbf{d}) \\
\text { s.t. } & P_{i}\left[g_{i}(\mathbf{X})>0\right] \leq 5 \% \quad i=1, \ldots, 3 \\
& d_{1} \in[2,7], \quad d_{2} \in[0.5,5.5] \\
& {\left[\sigma_{1}, \sigma_{2}\right]=[0.5,0.5],} \\
y(\mathbf{d})=- & \frac{\left(d_{1}+d_{2}-10\right)^{2}}{30}-\frac{\left(d_{1}-d_{2}+10\right)^{2}}{120}, \\
& g_{1}(\mathbf{X})=1-\frac{X_{1}^{2} X_{2}}{20},
\end{array}
$$

$$
\begin{aligned}
g_{2}(\mathbf{X})= & -1+\left(0.9063 X_{1}+0.4226 X_{2}-6\right)^{2} \\
& +\left(0.9063 X_{1}+0.4226 X_{2}-6\right)^{3} \\
& -0.6\left(0.9063 X_{1}+0.4226 X_{2}-6\right)^{4} \\
& -\left(-0.4226 X_{1}+0.9063 X_{2}\right) \\
& g_{3}(\mathbf{X})=1-\frac{80}{X_{1}^{2}+8 X_{2}+5}
\end{aligned}
$$

The problem and the optimal solution to the problem are graphically presented in Figure 10. It is expected that all three methods would have some instability due to the nature and formulation of the problem and the constraints. Table 3 summarizes the results for three approaches. 


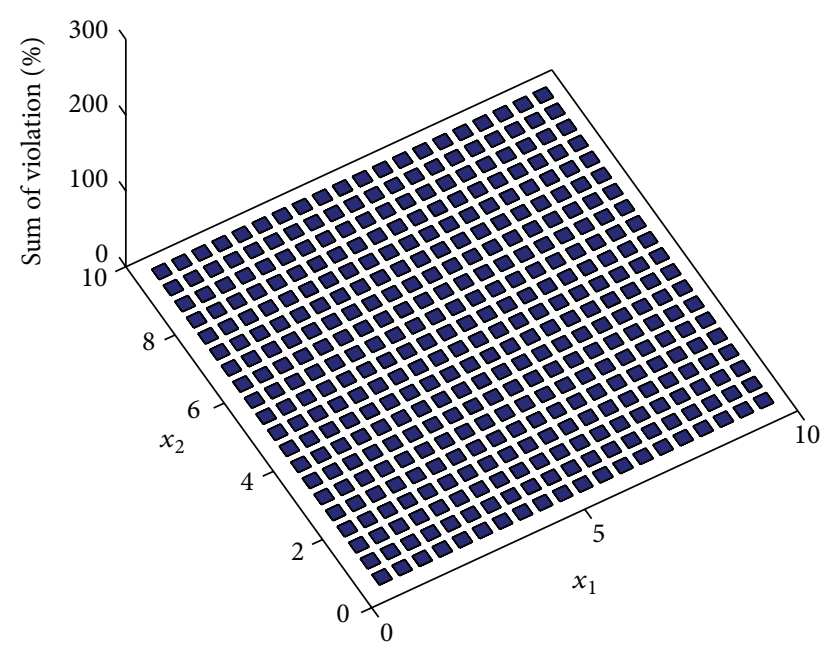

(a)

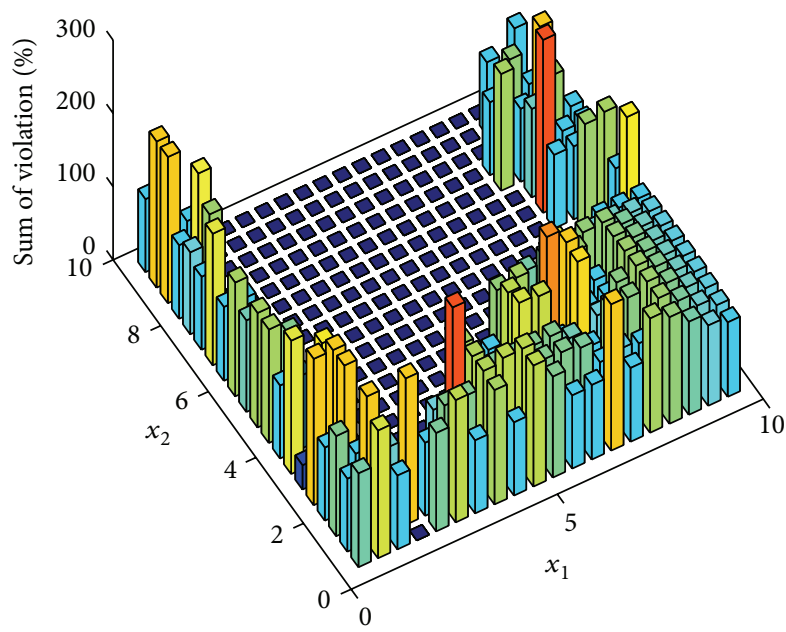

(b)

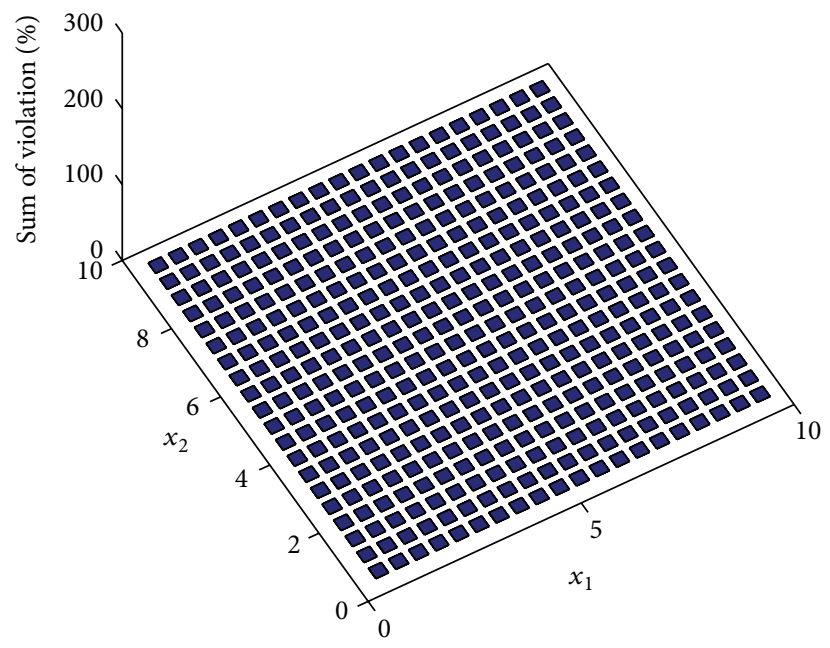

(c)

FIGURE 7: SOVs of the allowable probability of failure for 400 different starting points using (a) MRIA, (b) PMA, and (c) HRA.

In this problem, the PMA has the highest averaged SOV of $24.702 \%$, which was consistent with the first problem. The number of points with SOV $>1 \%$ was reported to be 118 points and some instances reported SOV s higher than $5 \%$. Incidence in PMA with SOVs higher than 5\% is presented in Figure 11.

Most of the instabilities come from regions with Cornell reliability indexes smaller than the allowable ones. In this case, PMA seems to perform poorly compared to the other approaches. The MRIA seems to be the most efficient given the constraints and the measure used for this paper. The HRA on the other hand performs better in terms of average FEs with the MRIA but is slightly higher on SOVs since it considers the PMA on instances where $S_{i} \leq 0$. The stability of HRA is somewhat at par to that of MRIA, especially when PMA suffers cases of numerical instabilities, such as these examples.

4.4. General Discussion of Presented Examples. Through these examples, the HRA proves to be a very useful approach to solve problems more stably using a few numbers of global iterations. This is useful when the nature of the problem is unknown or is constantly changing such as that in safety systems and complex products that would normally be numerically expensive to reach convergence. The stability of the solution using a low number of iterations is highlighted in this paper as presented in the numerical examples. The first nonlinear problem shows a general case for nonlinear problems; MRIA would yield stable results with efficiency expressed in terms of the FEs relatively poorer compared to PMA but PMA suffers from some instabilities which was also prominent in the highly nonlinear problem. The proposed HRA, on the other hand, shows stability close to that of MRIA with efficiencies close to that of PMA. The second nonlinear problem presents an example that highlights the efficiency of the PMA. In this case, the proposed HRA also encountered a slight drop in efficiency compared to the PMA. The highly nonlinear problem is an extreme case in which PMA suffers greatly in terms of the SOVs with some instances with SOVs 


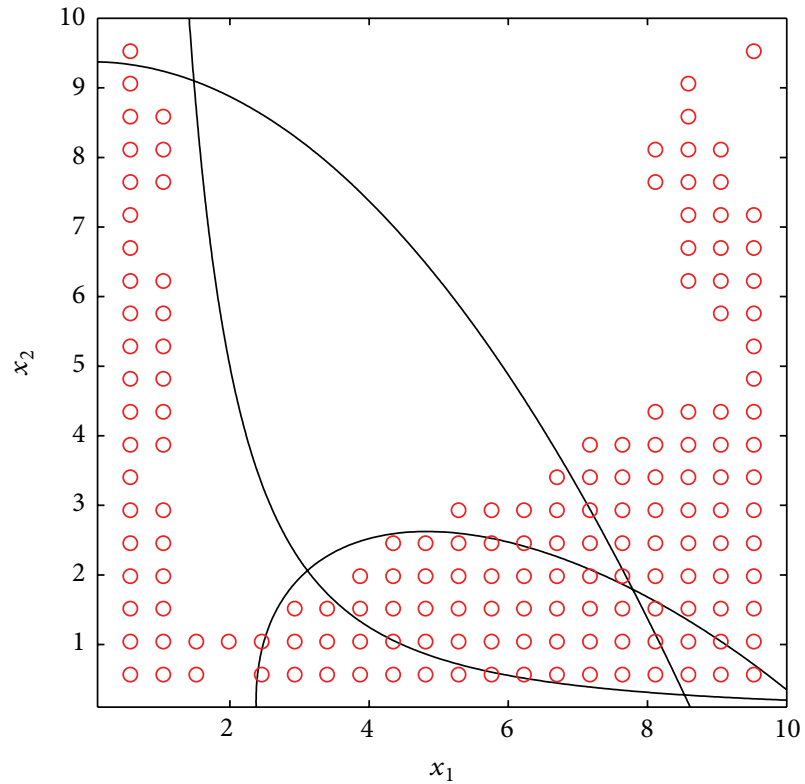

FIGURE 8: Location of starting points with SOV > 1\% using PMA.

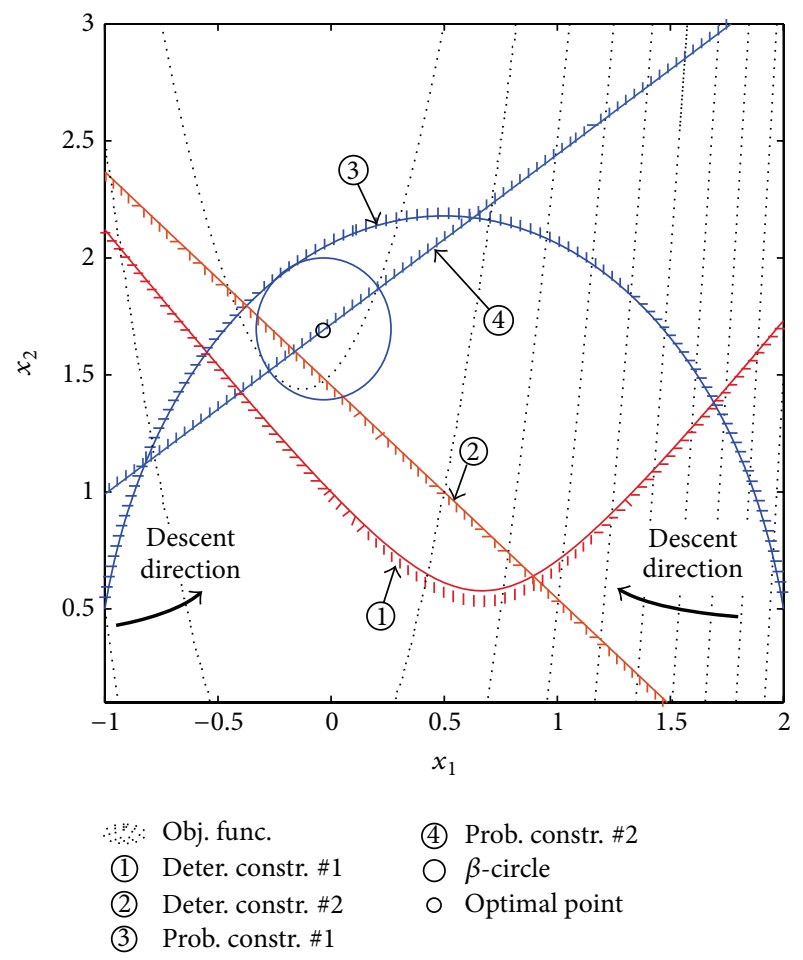

FIGURE 9: Graphical representation of Example 2.

higher than $5 \%$ as presented in Figure 11 . In this case, the MRIA remains stable with SOVs less than $2 \%$.

All of these problems were solved with 400 different starting points, which could represent a given design or operating condition for any system or component under the design space given highly irregular or unlikely constraints. The problems were chosen in such a way that they may be able

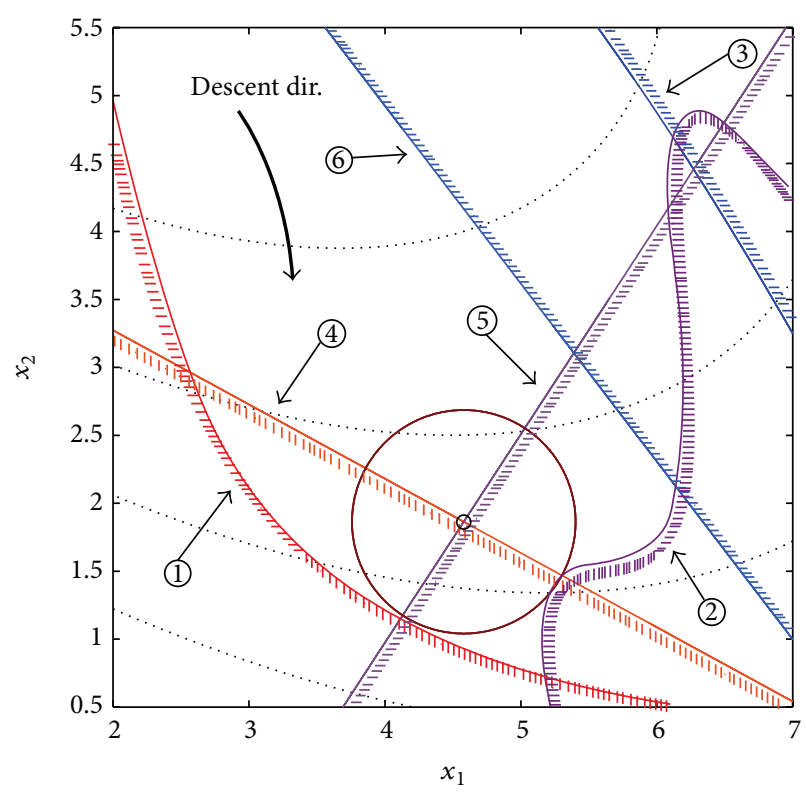
a: Obj. func.
(5) Prob. constr. \#2
(1) Deter. constr. \#1
(2) Deter. constr. \#2
(6) Prob. constr. \#3
(3) Deter. constr. \#3
○ $\beta$-Circle
(4) Prob. constr. \#1
Optimal point

FIGURE 10: Graphical representation of Example 3 [20].

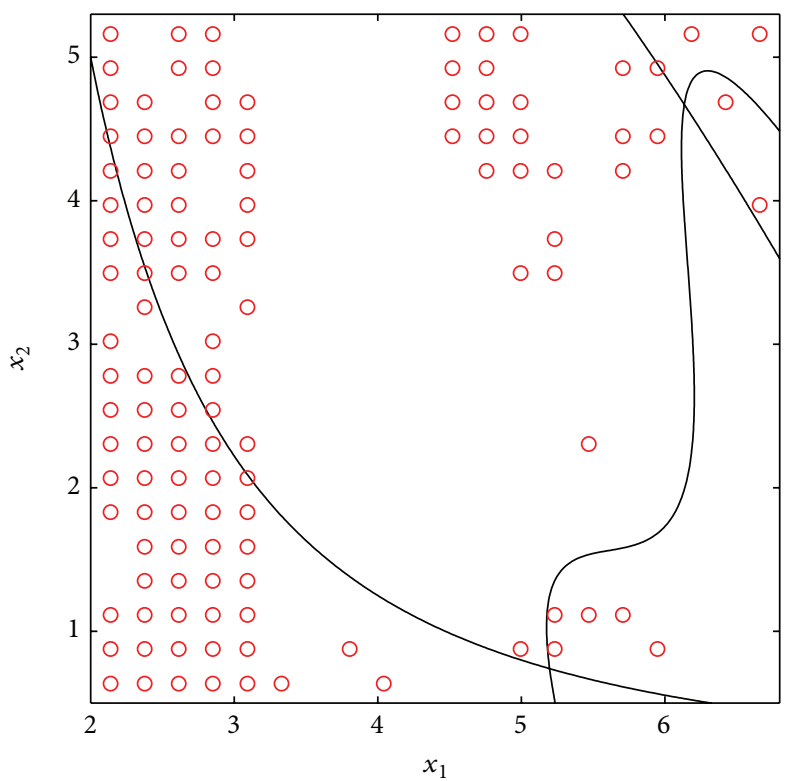

FIGURE 11: Location of starting points with SOV > 5\% using PMA.

to approximate extreme real world constraints for problems in which they are applied to. The number of global iterations was also limited to 5 . Keeping this constraint does not provide ample time for the methods to reach convergence but it is appropriate for problems under uncertainty or large complexity where computational time is expensive. The proposed HRA would always maintain a relatively stable result due to 
TABLE 3: FE and SOV of 400 starting points for Example 3.

\begin{tabular}{lccc}
\hline Approach & Average FE & Average SOV & $\begin{array}{c}\text { Number of } \\
\text { points with } \\
\text { high SOV }\end{array}$ \\
\hline MRIA & 282.7 & $1.694 \%$ & 10 \\
PMA & 371.4 & $24.702 \%$ & 118 \\
HRA & 245.3 & $1.783 \%$ & 13 \\
\hline
\end{tabular}

${ }^{*}$ Sum of violations $>1 \%$.

the nature of the MRIA formulation and efficiency is relatively faster compared to the MRIA due to the formulation of the PMA. Lastly, this approach could also be applicable to other approaches given that an appropriate selection factor for two or more approaches would be well established.

\section{Conclusion}

In summary, this paper proposes a hybrid reliability approach (HRA), which utilizes two popularly used RBDO algorithms, namely, the modified reliability index approach (MRIA) and the performance measure approach (PMA). HRA multifacetedly utilizes the stability of MRIA and the efficiency of PMA as exhibited by the presented nonlinear examples. The proposed HRA is novel in the selection factor and its formulation on the conditions on which method is going to be utilized. When the selection factor is smaller than or equal to zero, PMA is used. If the selection factor is greater than zero, the MRIA is used to formulate the probabilistic constraint until such time that the PMA becomes stable.

The three methods were then compared and contrasted under fixed iterations and starting points to three benchmark problems representing safety design and a highly nonlinear problem. It was proven that the method could never be worse in performance than PMA and MRIA. It was also demonstrated that the method has acquired the stability of MRIA and the efficiency of PMA.

\section{Nomenclature}

d: $\quad$ Expected value of $\mathbf{X} ; \mathbf{d}=E[\mathbf{X}]=d_{j} \mathbf{e}_{j}$ written in the Einstein notation

D: A threshold parameter

$\mathbf{e}_{j}$ : $\quad j$ th normal basis of the design variable

$f(\mathbf{x})$ : Joint probability density function of $\mathbf{X}$

$F_{g_{i}}$ : Cumulative distribution function (CDF) for the $i$ th constraint

$g_{i}: \quad i$ th constraint; $g_{i}(\mathbf{X})>0$ is considered as the failed design and $g_{i}(\mathbf{X}) \leq 0$ represents the feasible domain

$i$ : Dimensional index of the constraint; $i=1,2, \ldots, n$

$j: \quad$ Dimensional index of the design variable; $j=1,2, \ldots, N$

$k$ : Iteration index of the global loop in the RBDO process

$n$ : Number of the constraint
P: $\quad$ Probability of an event

$P_{f, i}: \quad$ Allowable failure probability with respect to the $i$ th constraint

$P_{\mathrm{MCS}, i}: i$ th failure probability based on Monte Carlo Simulations (MCS)

$S_{i}: \quad$ ith selection factor

$\mathbf{u}_{i}$ : Deterministic standard normal design variable associated with the $i$ th constraint; $\mathbf{u}_{i}=\sum_{j=1}^{N} u_{i, j} \mathbf{e}_{j}$ and $u_{i, j}=\sigma_{j}^{-1}\left(x_{j}-d_{j}\right) \forall i$

$\mathbf{U}_{i}$ : Random standard normal design variable for $i$ th constraint; $\mathbf{U}_{i}=\sum_{j=1}^{N} U_{i, j} \mathbf{e}_{j}$

$\mathbf{u}_{i}^{*}$ : $\quad$ Most probable failure point for $i$ th constraint; $\mathbf{u}_{i}^{*}=\sum_{j=1}^{N} u_{i, j}^{*} \mathbf{e}_{j}$

$\mathbf{u}_{i}^{\#}$ : $\quad$ Most probable target point for $i$ th constraint; $\mathbf{u}_{i}^{\#}=\sum_{j=1}^{N} u_{i, j}^{\#} \mathbf{e}_{j}$

$\mathbf{x}$ : Most probable failure point in the original design space; $\mathbf{x}^{*}=x_{j}^{*} \mathbf{e}_{j}$

$\mathbf{x}^{*}$ : $\quad$ Most probable target point in the original design space; $\mathbf{x}^{\#}=x_{j}^{\#} \mathbf{e}_{j}$

$\mathbf{x}^{\#}: \quad$ Deterministic design variable; $\mathbf{x}=x_{j} \mathbf{e}_{j}$

$\mathbf{X}: \quad$ Random design variable; $\mathbf{X}=X_{j} \mathbf{e}_{j}$

$y: \quad$ Cost function

$\beta_{f, i}: \quad$ Allowable modified reliability index for the $i$ th constraint

$\beta_{C, i}: \quad i$ th Cornell reliability index evaluated by mean-value first-order second-moment method

$\beta_{\mathrm{HL}, i}: \quad i$ th Hasofer-Lind reliability index

$\beta_{M, i}$ : Modified reliability index for the $i$ th constraint

$\gamma_{i}$ : $\quad$ Performance measure associated with the $i$ th constraint

$\boldsymbol{\sigma}: \quad$ Standard deviation of $\mathbf{X} ; \boldsymbol{\sigma}=\sum_{j=1}^{N} \sigma_{j} \mathbf{e}_{j} \mathbf{e}_{j}$

$\Phi: \quad$ Standard normal CDF.

\section{Conflict of Interests}

The authors declare that there is no conflict of interests regarding the publication of this paper.

\section{Acknowledgments}

The support from Institute of Nuclear Energy Research, Atomic Energy Council, Taiwan, and Chung Yuan Christian University, Taiwan, is greatly appreciated.

\section{References}

[1] B. R. Ellingwood, Development of a Probability Based Load Criterion for American National Standard A58: Building Code Requirements for Minimum Design Loads in Buildings and Other Structures, vol. 577, US Department of Commerce, National Bureau of Standards, Gaithersburg, Md, USA, 1980.

[2] S. S. Hasan, J. M. Leith, B. Campbell, R. Kapil, K. L. Smith, and F. A. Matsen III, "Characteristics of unsatisfactory shoulder arthroplasties," Journal of Shoulder and Elbow Surgery, vol. 11, no. 5, pp. 431-441, 2002. 
[3] R. D. Pope and R. A. Kramer, "Production uncertainty and factor demands for the competitive firm," Southern Economic Journal, vol. 48, no. 1, pp. 489-501, 1979.

[4] M. Karamouz and H. V. Vasiliadis, "Bayesian stochastic optimization of reservoir operation using uncertain forecasts," Water Resources Research, vol. 28, no. 5, pp. 1221-1232, 1992.

[5] H.-S. Jung and S. Cho, "Reliability-based topology optimization of geometrically nonlinear structures with loading and material uncertainties," Finite Elements in Analysis and Design, vol. 41, no. 3, pp. 311-331, 2004.

[6] E. Nikolaidis and R. Burdisso, "Reliability based optimization: a safety index approach," Computers and Structures, vol. 28, no. 6, pp. 781-788, 1988.

[7] I. Enevoldsen, "Reliability-based optimization as an information tool," Mechanics of Structures and Machines, vol. 22, no. 1, pp. 117-135, 1994.

[8] I. Enevoldsen and J. D. Sørensen, "Reliability-based optimization in structural engineering," Structural Safety, vol. 15, no. 3, pp. 169-196, 1994.

[9] S. V. L. Chandu and R. V. Grandhi, "General purpose procedure for reliability based structural optimization under parametric uncertainties," Advances in Engineering Software, vol. 23, no. 1, pp. 7-14, 1995.

[10] D. M. Frangopol and R. B. Corotis, "Reliability-based structural system optimization: state-of-the-art versus state-of-practice," in Analysis and Computation: Proceedings of the 12th Conference Held in Conjunction with Structures Congress XIV, F. Y. Cheng, Ed., pp. 67-78, 1996.

[11] Y. T. Wu and W. Wang, "A new method for efficient reliabilitybased design optimization," in Proceedings of the 7th Special Conference on Probabilistic Mechanics \& Structural Reliability, D. M. Frangopol and M. D. Grigoriu, Eds., pp. 274-277, 1996.

[12] A. D. S. Carter, Mechanical Reliability and Design, Wiley, New York, NY, USA, 1997.

[13] R. V. Grandhi and L. Wang, "Reliability-based structural optimization using improved two-point adaptive nonlinear approximations," Finite Elements in Analysis and Design, vol. 29, no. 1, pp. 35-48, 1998.

[14] J. Tu, K. K. Choi, and Y. H. Park, "A new study on reliabilitybased design optimization," Journal of Mechanical Design, vol. 121, no. 4, pp. 557-564, 1999.

[15] B. D. Youn and K. K. Choi, "An investigation of nonlinearity of reliability-based design optimization approaches," Journal of Mechanical Design, vol. 126, no. 3, pp. 403-411, 2004.

[16] B. D. Youn and K. K. Choi, "Selecting probabilistic approaches for realiability-based design optimization," AIAA Journal, vol. 42, no. 1, pp. 124-131, 2004.

[17] B. D. Youn, K. K. Choi, and L. Du, "Adaptive probability analysis using an enhanced hybrid mean value method," Structural and Multidisciplinary Optimization, vol. 29, no. 2, pp. 134-148, 2005.

[18] B. D. Youn, K. K. Choi, and L. Du, "Enriched performance measure approach for reliability-based design optimization," AIAA Journal, vol. 43, no. 4, pp. 874-884, 2005.

[19] P. T. Lin, H. C. Gea, and Y. Jaluria, "A modified reliability index approach for reliability-based design optimization," Journal of Mechanical Design, vol. 133, no. 4, Article ID 044501, 2011.

[20] P. T. Lin, Y. Jaluria, and H. C. Gea, "A hybrid reliability approach for reliability-based design optimization," in Proceedings of the ASME International Design Engineering Technical Conferences and Computers and Information in Engineering Conference (IDETC/CIE '10), pp. 1099-1107, Québec, Canada, August 2010.
[21] L. Gu, R. J. Yang, C. H. Tho, M. Makowski, O. Faruque, and Y. $\mathrm{Li}$, "Optimization and robustness for crashworthiness of side impact," International Journal of Vehicle Design, vol. 26, no. 4, pp. 348-360, 2001.

[22] K. J. Craig, N. Stander, D. A. Dooge, and S. Varadappa, "Automotive crashworthiness design using response surfacebased variable screening and optimization," Engineering Computations, vol. 22, no. 1, pp. 38-61, 2005.

[23] J.-F. Salavy, G. Aiello, P. Aubert et al., "Ferritic-Martensitic steel Test Blanket Modules: status and future needs for design criteria requirements and fabrication validation," Journal of Nuclear Materials, vol. 386-388, pp. 922-926, 2009.

[24] S. Syngellakis, Design against Blast: Load Definition \& Structural Response, Volume 11, WIT Press, 2013.

[25] R. J. Yang and L. Gu, "Experience with approximate reliabilitybased optimization methods," Structural and Multidisciplinary Optimization, vol. 26, no. 1-2, pp. 152-159, 2004.

[26] G. Cheng, L. Xu, and L. Jiang, "A sequential approximate programming strategy for reliability-based structural optimization," Computers and Structures, vol. 84, no. 21, pp. 1353-1367, 2006.

[27] H. C. Gea and K. Oza, "Two-level approximation method for reliability-based design optimisation," International Journal of Materials and Product Technology, vol. 25, no. 1-3, pp. 99-111, 2006.

[28] P. Yi, G. Cheng, and L. Jiang, "A sequential approximate programming strategy for performance-measure-based probabilistic structural design optimization," Structural Safety, vol. 30, no. 2, pp. 91-109, 2008.

[29] P. Yi and G. Cheng, "Further study on efficiency of sequential approximate programming for probabilistic structural design optimization," Structural and Multidisciplinary Optimization, vol. 35, no. 6, pp. 509-522, 2008.

[30] C. Onwubiko, Introduction to Engineering Design Optimization, Prentice Hall, Upper Saddle River, NJ, USA, 2000.

[31] A. M. Hasofer and N. C. Lind, "Exact and invariant secondmoment code format," Journal of the Engineering Mechanics Division, vol. 100, no. 1, pp. 111-121, 1974.

[32] R. Y. Rubinstein, Simulation and the Monte Carlo Method, Wiley, New York, NY, USA, 1981.

[33] K. Maute and D. M. Frangopol, "Reliability-based design of MEMS mechanisms by topology optimization," Computers and Structures, vol. 81, no. 8-11, pp. 813-824, 2003.

[34] L. Du and K. K. Choi, "An inverse analysis method for design optimization with both statistical and fuzzy uncertainties," Structural and Multidisciplinary Optimization, vol. 37, no. 2, pp. 107-119, 2008.

[35] B. D. Youn, K. K. Choi, and Y. H. Park, "Hybrid analysis method for reliability-based design optimization," Journal of Mechanical Design, vol. 125, no. 2, pp. 221-232, 2003.

[36] P. T. Lin, "Ensemble of unified reliability formulations (EURF)," in Proceedings of the 10th World Congress on Structural and Multidisciplinary Optimization (WCSMO '13), p. 5476, Orlando, Fla, USA, 2013.

[37] C. A. Cornell, "A probability-based structural code," Journal of the American Concrete Institute, vol. 66, no. 12, pp. 974-985, 1969.

[38] H. O. Madsen, S. Krenk, and N. C. Lind, Methods of Structural Safety, Prentice-Hall, Englewood Cliffs, NJ, USA, 1986.

[39] Y. T. Wu, O. H. Burnside, and T. A. Cruse, "Probabilistic methods for structural response analysis," in Computational 
Mechanics of Probabilistic and Reliability Analysis, W. K. Lam and T. Belytschko, Eds., pp. 181-196, Elme Press International, Lausanne, Switzerland.

[40] R. Rackwitz and B. Flessler, "Structural reliability under combined random load sequences," Computers and Structures, vol. 9, no. 5, pp. 489-494, 1978.

[41] Y. T. Wu, Y. Shin, R. Sues, and M. Cesare, "Safety-factor based approach for probability-based design optimization," in Proceedings of the 2nd AIAA/ASME/ASCE/AHS/ASC Structures, Structural Dynamics and Materials Conference and Exhibit, Seattle, Wash, USA, 2001.

[42] I. Lee, K. K. Choi, L. Du, and D. Gorsich, "Inverse analysis method using MPP-based dimension reduction for reliabilitybased design optimization of nonlinear and multi-dimensional systems," Computer Methods in Applied Mechanics and Engineering, vol. 198, no. 1, pp. 14-27, 2008.

[43] X. Du and W. Chen, "Sequential optimization and reliability assessment method for efficient probabilistic design," Journal of Mechanical Design, vol. 126, no. 2, pp. 225-233, 2004. 


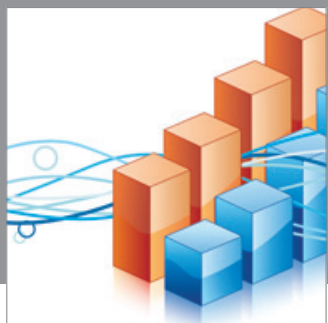

Advances in

Operations Research

mansans

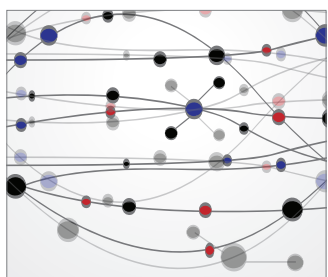

The Scientific World Journal
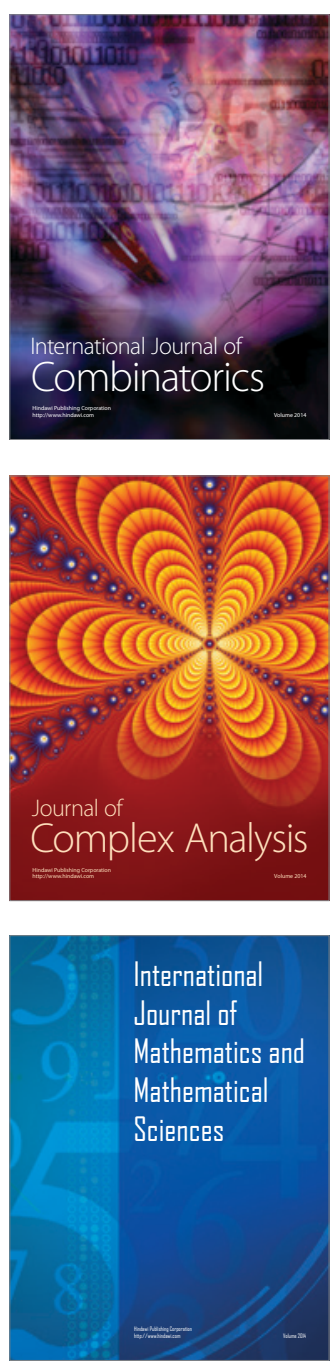
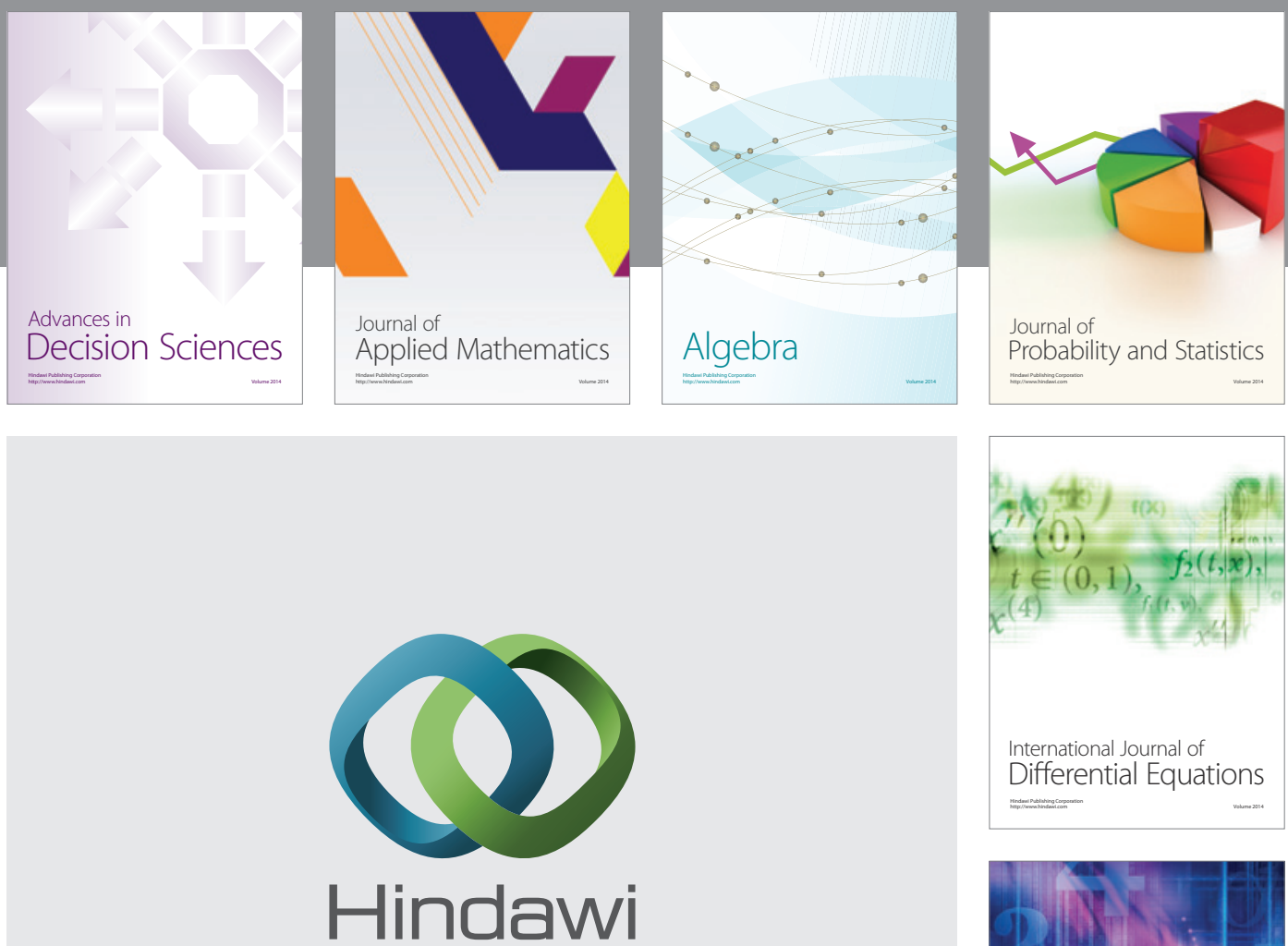

Submit your manuscripts at http://www.hindawi.com
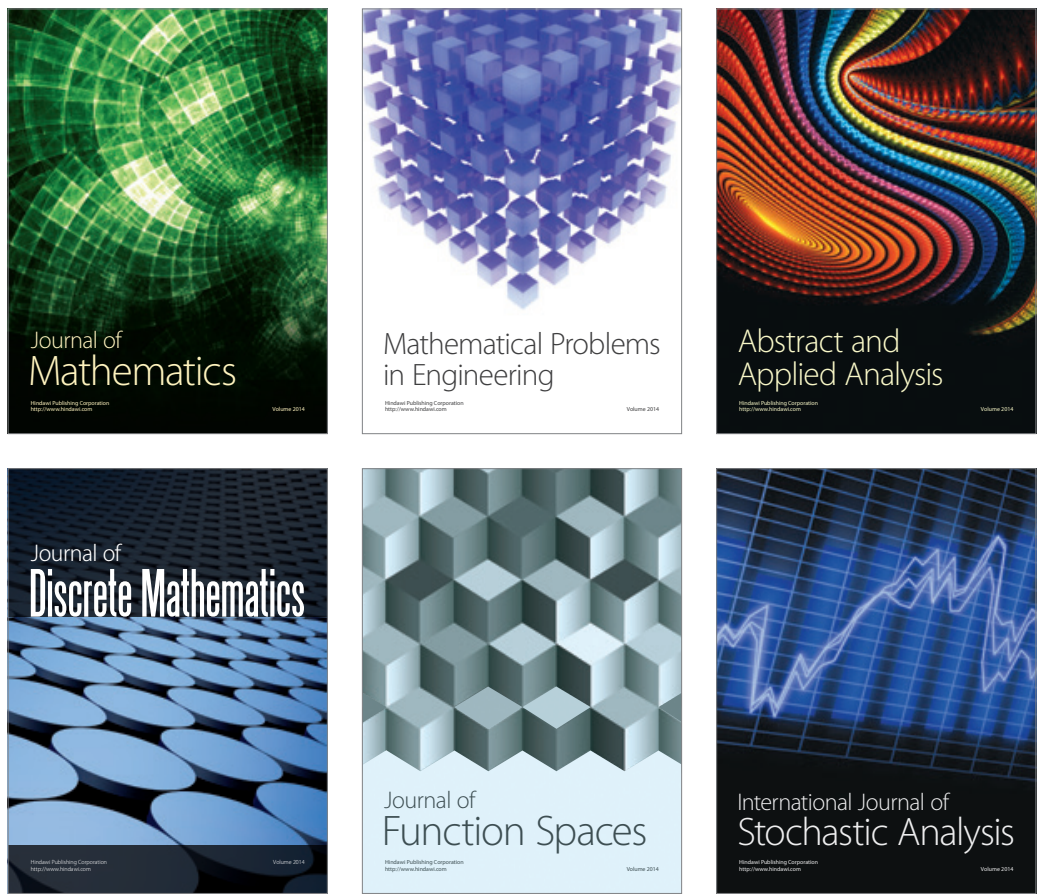

Journal of

Function Spaces

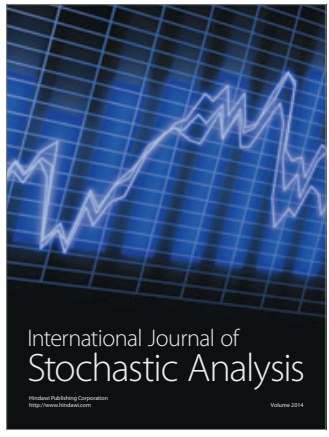

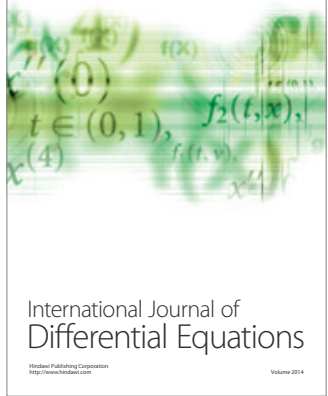
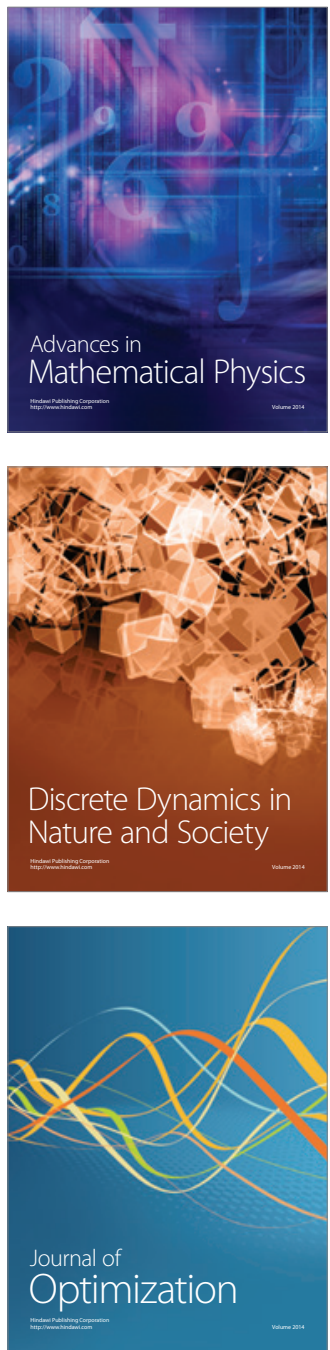